\title{
Sums of Reciprocals of Triple Binomial Coefficients
}

\author{
A. Sofo \\ School of Computer Science and Mathematics, Victoria University, P.O. Box 14428, Melbourne, \\ VIC 8001, Australia
}

Correspondence should be addressed to A. Sofo, anthony.sofo@vu.edu.au

Received 28 August 2007; Accepted 17 December 2007

Recommended by George Andrews

We investigate the integral representation of infinite sums involving the reciprocals of triple binomial coefficients. We also recover some wellknown properties of $\zeta(3)$ and extend the range of results given by other authors.

Copyright (C) 2008 A. Sofo. This is an open access article distributed under the Creative Commons Attribution License, which permits unrestricted use, distribution, and reproduction in any medium, provided the original work is properly cited.

\section{Introduction}

In this paper, we investigate the summation of the reciprocal of triple products of combinatorial coefficients. In particular, we develop integral representations for

$$
\sum_{n=0}^{\infty} \frac{1}{\left(\begin{array}{c}
a n+j \\
j
\end{array}\right)\left(\begin{array}{c}
b n+k \\
k
\end{array}\right)\left(\begin{array}{c}
c n+l \\
l
\end{array}\right)}, \quad \sum_{n=0}^{\infty} \frac{\left(\begin{array}{c}
n+m-1 \\
n
\end{array}\right)}{\left(\begin{array}{c}
a n+j \\
j
\end{array}\right)\left(\begin{array}{c}
b n+k \\
k
\end{array}\right)\left(\begin{array}{c}
c n+l \\
l
\end{array}\right)}
$$

and their alternating series counterparts.

For the representation of sums of reciprocals of single and double binomial coefficients, one may refer to some results in the papers [1-3], see also [4].

For designated cases of the parameter values $(a, b, c, j, k, l, m)$, various particular sums may be expressed in terms of $\zeta(2)$ and $\zeta(3)$. For many interesting properties of the Zeta function, the reader is referred to [5].

The representation of sums in terms of integrals is extremely useful because it allows one to estimate bounds on the sums in cases they cannot be written in closed form. Convexity properties for sums may also be investigated.

Apéry's [6], see also Beukers [7], proof of the irrationality of $\zeta(3)$ uses an elementary and quite complicated construction of the approximants $\alpha_{n} / \beta_{n} \in Q$ to this number based on 
a recurrence relation. The integral representation

$$
\iiint_{0}^{1} \frac{\{x(1-x) y(1-y) z(1-z)\}^{n}}{(1-(1-x y) z)^{n+1}} d x d y d z=2 \beta_{n} \zeta(3)-2 \alpha_{n}
$$

for the sequence $\left\{\alpha_{n}, \beta_{n}\right\}$ was proposed.

It is important to note that other integral representations of $\zeta(3)$ are available in terms of both single and double integrals. Guillera and Sondow [8] list a number of them including the classical results

$$
\begin{aligned}
& \iint_{0}^{1} \frac{-\ln (x y)}{1-x y} d x d y=2 \zeta(3), \\
& \iint_{0}^{1} \frac{\ln (2-x y)}{1-x y} d x d y=\frac{5}{8} \zeta(3) .
\end{aligned}
$$

In a recent paper, Muzaffar [9] also obtained some results of the combinatorial type

$$
\sum_{n=0}^{\infty} \frac{\left(\begin{array}{c}
2 n \\
n
\end{array}\right)}{\left(\begin{array}{c}
2 n+1 \\
1
\end{array}\right)\left(\begin{array}{c}
2 n+k+1 \\
1
\end{array}\right)\left(\begin{array}{c}
2 n+2 k \\
n+k
\end{array}\right)}=\alpha_{k} \pi^{2}+\beta_{k}
$$

by utilising the power series expansion of $\left(\sin ^{-1} x\right)^{q}$, and $\left(\alpha_{k}, \beta_{k}\right)$ are constants depending on $k \geq 0$. In this paper, we complement and extend some of the results given by Muzaffar.

There are some identities in the literature involving reciprocals of triple products of combinatorial coefficients, one prominentidentity is the Dougall identity, see [10] or [11],

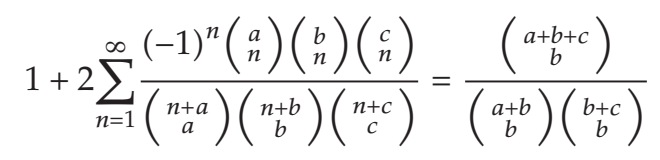

for $R(a+b+c)>-1$.

\section{The main results}

In this section, we develop integral identities for reciprocals of triple products of binomial coefficients.

Theorem 2.1. For $a, b$, and $c$ positive real numbers and $j, k, l \geq 0$, then

$$
\begin{aligned}
S(a, b, c, j, k, l) & =\sum_{n=0}^{\infty} \frac{1}{\left(\begin{array}{c}
a n+j \\
j
\end{array}\right)\left(\begin{array}{c}
b n+k \\
k
\end{array}\right)\left(\begin{array}{c}
c n+l \\
l
\end{array}\right)} \\
& =j k l \iiint_{0}^{1} \frac{(1-x)^{j-1}(1-y)^{k-1}(1-z)^{l-1}}{1-X Y Z} d x d y d z
\end{aligned}
$$


A. Sofo

$$
\begin{aligned}
& =1+a b c \iiint_{0}^{1} \frac{(1-x)^{j}(1-y)^{k}(1-z)^{l} X Y Z\left((X Y Z)^{2}+4 X Y Z+1\right) d x d y d z}{x y z(1-X Y Z)^{4}}
\end{aligned}
$$

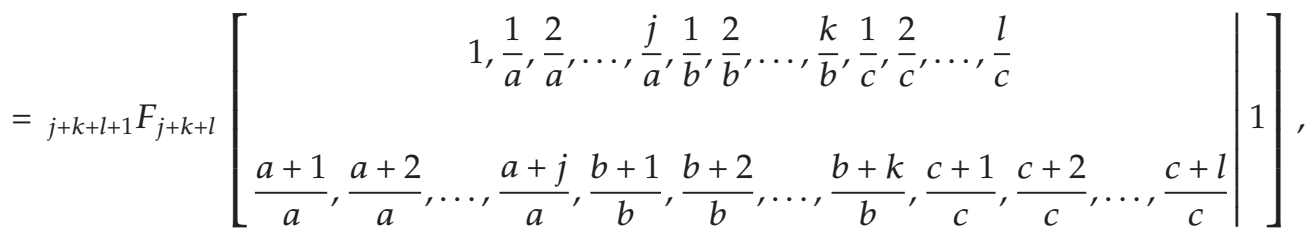

and similarly

$$
\begin{aligned}
& T(a, b, c, j, k, l) \\
& =\sum_{n=0}^{\infty} \frac{(-1)^{n}}{\left(\begin{array}{c}
a n+j \\
j
\end{array}\right)\left(\begin{array}{c}
b n+k \\
k
\end{array}\right)\left(\begin{array}{c}
c n+l \\
l
\end{array}\right)} \\
& =j k l \iiint_{0}^{1} \frac{(1-x)^{j-1}(1-y)^{k-1}(1-z)^{l-1}}{1+X Y Z} d x d y d z \\
& =1-a b c \iiint_{0}^{1} \frac{(1-x)^{j}(1-y)^{k}(1-z)^{l} X Y Z\left((X Y Z)^{2}-4 X Y Z+1\right) d x d y d z}{x y z(1+X Y Z)^{4}} \\
& ={ }_{j+k+l+1} F_{j+k+l}\left[\frac{a+1}{a}, \frac{a+2}{a}, \ldots, \frac{a+j}{a}, \frac{b+1}{b}, \frac{j}{a}, \frac{1}{b}, \frac{2}{b}, \ldots, \frac{k}{b}, \frac{1}{c}, \frac{2}{c}, \ldots, \frac{l}{c}, \ldots, \frac{b+k}{b}, \frac{c+1}{c}, \frac{c+2}{c}, \ldots, \frac{c+l}{c} \mid-1\right]
\end{aligned}
$$

where

$$
X Y Z=x^{a} y^{b} z^{c} .
$$

Proof. Consider (2.1):

$$
\begin{aligned}
S(a, b, c, j, k, l) & =\sum_{n=0}^{\infty} \frac{1}{\left(\begin{array}{c}
a n+j \\
j
\end{array}\right)\left(\begin{array}{c}
b n+k \\
k
\end{array}\right)\left(\begin{array}{c}
c n+l \\
l
\end{array}\right)} \\
& =j k l \sum_{n=0}^{\infty} \frac{\Gamma(a n+1) \Gamma(j) \Gamma(b n+1) \Gamma(k) \Gamma(c n+1) \Gamma(l)}{\Gamma(a n+j+1) \Gamma(b n+k+1) \Gamma(c n+l+1)} \\
& =j k l \sum_{n=0}^{\infty} B(a n, j+1) B(b n, k+1) B(c n, l+1),
\end{aligned}
$$


where $\Gamma(\cdot)$ is the classical Gamma function and $B(\cdot, \cdot)$ is the Beta function. It holds that

$$
\begin{aligned}
S(a, b, c, j, k, l) & =j k l \sum_{n=0}^{\infty} \int_{x=0}^{1}(1-x)^{j-1} x^{a n} d x \int_{y=0}^{1}(1-y)^{k-1} y^{b n} d y \int_{z=0}^{1}(1-z)^{l-1} z^{c n} d z \\
& =j k l \int_{x=0}^{1} \int_{y=0}^{1} \int_{z=0}^{1}(1-x)^{j-1}(1-y)^{k-1}(1-z)^{l-1} \sum_{n=0}^{\infty}\left(x^{a} y^{b} z^{c}\right)^{n} d x d y d z
\end{aligned}
$$

by an allowable change of integral and sum, and hence we have

$$
S(a, b, c, j, k, l)=j k l \iiint_{0}^{1} \frac{(1-x)^{j-1}(1-y)^{k-1}(1-z)^{l-1}}{1-X Y Z} d x d y d z
$$

which is the result (2.2).

To prove identity (2.3), consider (2.1) and expand as follows:

$$
\begin{aligned}
& S(a, b, c, j, k, l) \\
& \quad=\sum_{n=0}^{\infty} \frac{a b c n^{3} \Gamma(a n) \Gamma(j+1) \Gamma(b n) \Gamma(k+1) \Gamma(c n) \Gamma(l+1)}{\Gamma(a n+j+1) \Gamma(b n+k+1) \Gamma(c n+l+1)} \\
& \quad=\sum_{n=0}^{\infty} a b c n^{3} B(j+1, a n) B(k+1, b n) B(k+1, c n) \\
& =1+a b c \iiint_{0}^{1} \frac{(1-x)^{j}(1-y)^{k}(1-z)^{l}}{x y z} \sum_{n=1}^{\infty} n^{3}\left(x^{a} y^{b} z^{c}\right)^{n} d x d y d z \\
& =1+a b c \iiint_{0}^{1} \frac{(1-x)^{j}(1-y)^{k}(1-z)^{l}}{x y z(1-X Y Z)^{4}} X Y Z\left((X Y Z)^{2}+4 X Y Z+1\right) d x d y d z
\end{aligned}
$$

which is the result (2.3). The results (2.6) and (2.7) may be obtained in a similar fashion and therefore will not be pursued here.

The hypergeometric representation (2.4) and (2.8) can be obtained by the consideration of the ratio of successive terms (2.1) and (2.5), respectively.

We may also note that from known properties of the hypergeometric function, we may write, from (2.4) and (2.8),

$$
{ }_{j+k+l+1} F_{j+k+l}\left[\begin{array}{c}
1, \frac{1}{a}, \frac{2}{a}, \ldots, \frac{j}{a}, \frac{1}{b}, \frac{2}{b}, \ldots, \frac{k}{b}, \frac{1}{c}, \frac{2}{c}, \ldots, \frac{l}{c} \\
\frac{a+1}{a}, \frac{a+2}{a}, \ldots, \frac{a+j}{a}, \frac{b+1}{b}, \frac{b+2}{b}, \ldots, \frac{b+k}{b}, \frac{c+1}{c}, \frac{c+2}{c}, \ldots, \frac{c+l}{c} \mid
\end{array}\right]
$$




$$
\begin{aligned}
& \left.=a_{a+b+c+1} F_{a+b+c}\left[\begin{array}{c}
1,1,1,1, \frac{1}{a}, \frac{2}{a}, \ldots, \frac{a-1}{a}, \frac{1}{b}, \frac{2}{b}, \ldots, \frac{b-1}{b}, \frac{1}{c}, \frac{2}{c}, \ldots, \frac{c-1}{c} \\
\frac{j+1}{a}, \frac{j+2}{a}, \ldots, \frac{j+a}{a}, \frac{k+1}{b}, \frac{k+2}{b}, \ldots, \frac{k+b}{b}, \frac{l+1}{c}, \frac{l+2}{c}, \ldots, \frac{l+c}{c}
\end{array}\right] 1\right] \\
& \left.j+k+l+1 F_{j+k+l}\left[\begin{array}{c}
1, \frac{1}{a}, \frac{2}{a}, \ldots, \frac{j}{a}, \frac{1}{b}, \frac{2}{b}, \ldots, \frac{k}{b}, \frac{1}{c}, \frac{2}{c}, \ldots, \frac{l}{c} \\
\frac{a+1}{a}, \frac{a+2}{a}, \ldots, \frac{a+j}{a}, \frac{b+1}{b}, \frac{b+2}{b}, \ldots, \frac{b+k}{b}, \frac{c+1}{c}, \frac{c+2}{c}, \ldots, \frac{c+l}{c}
\end{array}\right]-1\right] \\
& \left.={ }_{a+b+c+1} F_{a+b+c}\left[\begin{array}{c}
1,1,1,1, \frac{1}{a}, \frac{2}{a}, \ldots, \frac{a-1}{a}, \frac{1}{b}, \frac{2}{b}, \ldots, \frac{b-1}{b}, \frac{1}{c}, \frac{2}{c}, \ldots, \frac{c-1}{c} \\
\frac{j+1}{a}, \frac{j+2}{a}, \ldots, \frac{j+a}{a}, \frac{k+1}{b}, \frac{k+2}{b}, \ldots, \frac{k+b}{b}, \frac{l+1}{c}, \frac{l+2}{c}, \ldots, \frac{l+c}{c}
\end{array}\right]-1\right]
\end{aligned}
$$

\section{Examples}

Example 3.1. It holds that

$$
\begin{aligned}
S(1,1,1,1,1,1) & =\sum_{n=0}^{\infty} \frac{1}{(n+1)^{3}}=\zeta(3)=\iiint_{0}^{1} \frac{d x d y d z}{1-x y z}={ }_{4} F_{3}\left[\begin{array}{c}
1,1,1,1 \\
2,2,2
\end{array} \mid 1\right] \\
& =1+\iiint_{0}^{1} \frac{(1-x)(1-y)(1-z)\left((x y z)^{2}+4 x y z+1\right)}{(1-x y z)^{4}} d x d y d z .
\end{aligned}
$$

Other integral representations of $\zeta(3)$ do exist, some of which are as follows.

Finch [12] gave the expression

$$
(-1)^{n} n ! \zeta(n+1)=\int_{x=0}^{1} \frac{\{\ln (x)\}^{n}}{1-x} d x=\int_{x=0}^{1} \frac{\{\ln (1-x)\}^{n}}{x} d x .
$$

Lord [13] posed the problem to show that

$$
\begin{aligned}
S & (2,2,2,1,1,1) \\
& =\sum_{n=0}^{\infty} \frac{1}{(2 n+1)^{3}}=\frac{7}{8} \zeta(3)=\int_{x=0}^{\pi / 4} \frac{\ln (\cos (x)) \ln (\sin (x))}{\cos (x) \sin (x)} d x=\iiint_{0}^{1} \frac{d x d y d z}{1-x^{2} y^{2} z^{2}} \\
& =1+8 \iiint_{0}^{1} \frac{(1-x)(1-y)(1-z) x y z\left((x y z)^{4}+4(x y z)^{2}+1\right)}{\left(1-x^{2} y^{2} z^{2}\right)^{4}} d x d y d z \\
& ={ }_{4} F_{3}\left[\begin{array}{c}
\frac{1}{2}, \frac{1}{2}, \frac{1}{2}, 1 \\
\frac{3}{2}, \frac{3}{2}, \frac{3}{2}
\end{array} \mid 1\right],
\end{aligned}
$$

the last three expressions are directly from (2.2), (2.3), and (2.4), respectively. 
Nan-Yue and Williams [14] also gave

$$
\zeta(3)=-5 \int_{x=0}^{\ln \left(\phi^{2}\right)} x \ln \left(2 \sinh \left(\frac{x}{2}\right)\right) d x
$$

where $\phi=$ golden ratio $=(1+\sqrt{5}) / 2$.

Example 3.2. It holds that

$$
\begin{aligned}
S(4,2,3, j, k, l) & =\sum_{n=0}^{\infty} \frac{1}{\left(\begin{array}{c}
4 n+j \\
j
\end{array}\right)\left(\begin{array}{c}
2 n+k \\
k
\end{array}\right)\left(\begin{array}{c}
3 n+l \\
l
\end{array}\right)} \\
& =\sum_{n=0}^{\infty} \frac{j ! k ! l !}{\prod_{r=1}^{j}(4 n+r) \prod_{r=1}^{k}(2 n+r) \prod_{r=1}^{l}(3 n+r)} \\
& =j k l \iiint_{0}^{1} \frac{(1-x)^{j-1}(1-y)^{k-1}(1-z)^{l-1} d x d y d z}{1-x^{4} y^{2} z^{3}} \\
& ={ }_{10} F_{9}\left[\frac{j+1}{4}, \frac{j+2}{4}, \frac{j+3}{4} \frac{j+4}{4}, \frac{k+1}{2}, \frac{3}{4}, \frac{2}{3}, \frac{1}{2}, \frac{1}{2}, \frac{1}{3}, \frac{1}{4}, \frac{l+1}{3}, \frac{l+2}{3}, \frac{l+3}{3} \mid 1\right] \\
& =\alpha_{1}+\alpha_{2} \pi+\alpha_{3} \zeta(2)+\alpha_{4} \ln (2)+\alpha_{5} \ln (3)+\alpha_{6} \zeta(3) .
\end{aligned}
$$

For $j=5, k=6$, and $l=6$, we have the values

$$
\begin{aligned}
& \alpha_{1}=\frac{495762799093}{17 \cdot 11 \cdot 7^{2} \cdot 3^{2} \cdot 2^{4}}, \quad \alpha_{2}=\left(\frac{167 \cdot 2^{25}}{17 \cdot 13 \cdot 11 \cdot 7^{2} \cdot 3^{2}}-\frac{23 \cdot 5 \cdot 3^{16} \sqrt{3}}{17 \cdot 13 \cdot 11 \cdot 7^{2} \cdot 2^{4}}\right), \\
& \alpha_{3}=\frac{1709 \cdot 5 \cdot 2}{7}, \quad \alpha_{4}=-\frac{755357 \cdot 2^{19}}{17 \cdot 13 \cdot 11 \cdot 7^{2} \cdot 3^{2}}, \quad \alpha_{5}=\frac{43 \cdot 23 \cdot 5 \cdot 3^{16}}{17 \cdot 13 \cdot 11 \cdot 7^{2} \cdot 2^{4}}, \quad \alpha_{6}=5^{3} \cdot 3 \cdot 2^{2} .
\end{aligned}
$$

Example 3.3. For the alternating case,

$$
\begin{aligned}
T(1,1,1,1,1,1) & =\sum_{n=0}^{\infty} \frac{(-1)^{n}}{(n+1)^{3}}=\frac{3}{4} \zeta(3) \\
& =\iiint_{0}^{1} \frac{d x d y d z}{1+x y z}={ }_{4} F_{3}\left[\begin{array}{c}
1,1,1,1 \\
2,2,2
\end{array} \mid-1\right] \\
& =1-\iiint_{0}^{1} \frac{(1-x)(1-y)(1-z)\left((x y z)^{2}-4 x y z+1\right)}{(1+x y z)^{4}} d x d y d z .
\end{aligned}
$$


Example 3.4. It holds that

$$
\begin{aligned}
& T(2 / 3,2 / 3,5 / 6,2,4,3) \\
& =\sum_{n=0}^{\infty} \frac{(-1)^{n}}{\left(\begin{array}{c}
2 n / 3+2 \\
2
\end{array}\right)\left(\begin{array}{c}
2 n / 3+4 \\
4
\end{array}\right)\left(\begin{array}{c}
5 n / 6+3 \\
3
\end{array}\right)} \\
& =\sum_{n=0}^{\infty} \frac{(-1)^{n} 2^{5} 3^{11}}{(n+3)^{2}(n+6)(2 n+3)^{2}(2 n+9)(5 n+6)(5 n+12)(5 n+18)} \\
& =24 \iiint_{0}^{1} \frac{(1-x)(1-y)^{3}(1-z)^{2} d x d y d z}{1+x^{2 / 3} y^{2 / 3} z^{5 / 6}} \\
& \left.={ }_{10} F_{9}\left[\begin{array}{l}
1, \frac{3}{2}, \frac{3}{2}, 3,3, \frac{9}{2}, 6, \frac{6}{5}, \frac{12}{5}, \frac{18}{5} \\
\frac{5}{2}, \frac{5}{2}, 4,4, \frac{11}{2}, \frac{11}{5}, \frac{17}{5}, \frac{23}{5}, 7
\end{array}\right]-1\right] \\
& =\frac{70663 \cdot 1669}{13 \cdot 11 \cdot 7 \cdot 5 \cdot 3 \cdot 2}+3^{3} \cdot 2^{4} \zeta(2)+\frac{3^{2} \cdot 2^{10}}{7} G+\frac{109 \cdot 5 \cdot 3 \cdot 2^{9}}{11 \cdot 7^{2}} \ln 2-\frac{2^{8} \cdot 3^{4}}{11 \cdot 7^{2}} \pi \\
& +\left(\frac{113 \cdot 5^{6} \cdot \sqrt{5}}{11 \cdot 7^{2} \cdot 2^{2}}-\frac{271 \cdot 5^{5}}{11 \cdot 7^{2} \cdot 2^{3}}\right) \ln (\alpha)-\left(\frac{113 \cdot 5^{6} \cdot \sqrt{5}}{11 \cdot 7^{2} \cdot 2^{2}}+\frac{271 \cdot 5^{5}}{11 \cdot 7^{2} \cdot 2^{3}}\right) \ln (\phi) \\
& -\left(\frac{5^{4} \cdot 3 \cdot \sqrt{5} \cdot \phi \sqrt{\alpha \sqrt{5}}}{11 \cdot 2}+\frac{37 \cdot 5^{4} \cdot \sqrt{5} \cdot \alpha \sqrt{\phi \sqrt{5}}}{7^{2}}\right) \pi
\end{aligned}
$$

where $G$ is Catalan's constant, $\phi$ is the golden ratio, and $\alpha=$ silver ratio $=(\sqrt{5}-1) / 2$.

Now consider the following theorem, which is a generalisation of Theorem 2.1.

Theorem 3.5. For $a, b, c$, and $m$ positive real numbers and $j, k, l \geq 0$ with $j+k+l \geq m$, then

$$
\begin{aligned}
& Q(a, b, c, j, k, l, m) \\
& \quad=\sum_{n=0}^{\infty} \frac{\left(\begin{array}{c}
n+m-1 \\
n
\end{array}\right)}{\left(\begin{array}{c}
a+j \\
j
\end{array}\right)\left(\begin{array}{c}
b n+k \\
k
\end{array}\right)\left(\begin{array}{c}
c n+l \\
l
\end{array}\right)} \\
& \quad=\sum_{n=0}^{\infty} \frac{j ! k ! l !(n+1)_{m-1}}{(m-1) !(a n+1)_{j}(b n+1)_{k}(c n+1)_{l}} \\
& \quad=j k l \iiint_{0}^{1} \frac{(1-x)^{j-1}(1-y)^{k-1}(1-z)^{l-1}}{(1-X Y Z)^{m}} d x d y d z
\end{aligned}
$$




$$
\begin{aligned}
& =1+m a b c \iiint_{0}^{1} \frac{(1-x)^{j}(1-y)^{k}(1-z)^{l} X Y Z\left((X Y Z)^{2}+(3 m+1) X Y Z+1\right) d x d y d z}{x y z(1-X Y Z)^{m+3}} \\
& ={ }_{j+k+l+1} F_{j+k+l}\left[\begin{array}{c}
m, \frac{1}{a}, \frac{2}{a}, \ldots, \frac{j}{a}, \frac{1}{b}, \frac{2}{b}, \ldots, \frac{k}{b}, \frac{1}{c}, \frac{2}{c}, \ldots, \frac{l}{c} \\
\left.\frac{a+1}{a}, \frac{a+2}{a}, \ldots, \frac{a+j}{a}, \frac{b+1}{b}, \frac{b+2}{b}, \ldots, \frac{b+k}{b}, \frac{c+1}{c}, \frac{c+2}{c}, \ldots, \frac{c+l}{c} \mid 1\right],
\end{array}\right.
\end{aligned}
$$

$$
\begin{aligned}
& R(a, b, c, j, k, l, m) \\
& =\sum_{n=0}^{\infty} \frac{(-1)^{n}\left(\begin{array}{c}
n+m-1 \\
n
\end{array}\right)}{\left(\begin{array}{c}
a n+j \\
j
\end{array}\right)\left(\begin{array}{c}
b n+k \\
k
\end{array}\right)\left(\begin{array}{c}
c n+l \\
l
\end{array}\right)} \\
& =\sum_{n=0}^{\infty} \frac{(-1)^{n} j ! k ! l !(n+1)_{m-1}}{(m-1) !(a n+1)_{j}(b n+1)_{k}(c n+1)_{l}} \\
& =j k l \iiint_{0}^{1} \frac{(1-x)^{j-1}(1-y)^{k-1}(1-z)^{l-1}}{(1+X Y Z)^{m}} d x d y d z \\
& =1-m a b c \iiint_{0}^{1} \frac{(1-x)^{j}(1-y)^{k}(1-z)^{l} X Y Z\left((X Y Z)^{2}-(3 m+1) X Y Z+1\right) d x d y d z}{x y z(1+X Y Z)^{m+3}} \\
& ={ }_{j+k+l+1} F_{j+k+l}\left[\begin{array}{c}
m, \frac{1}{a}, \frac{2}{a}, \ldots, \frac{j}{a}, \frac{1}{b}, \frac{2}{b}, \ldots, \frac{k}{b}, \frac{1}{c}, \frac{2}{c}, \ldots, \frac{l}{c} \\
\frac{a+1}{a}, \frac{a+2}{a}, \ldots, \frac{a+j}{a}, \frac{b+1}{b}, \frac{b+2}{b}, \ldots, \frac{b+k}{b}, \frac{c+1}{c}, \frac{c+2}{c}, \ldots, \frac{c+l}{c} \mid-1
\end{array}\right],
\end{aligned}
$$

where XYZ is given by (2.9) and

$$
(p)_{\alpha}=p(p+1) \cdots(p+\alpha-1)=\frac{\Gamma(p+\alpha)}{\Gamma(p)}
$$

is Pochhammer's symbol. 
Proof. Consider (3.14):

$$
\begin{aligned}
& R(a, b, c, j, k, l, m) \\
& \quad=\sum_{n=0}^{\infty} \frac{(-1)^{n}\left(\begin{array}{c}
n+m-1 \\
n
\end{array}\right)}{\left(\begin{array}{c}
a n+j \\
j
\end{array}\right)\left(\begin{array}{c}
c n+k \\
k
\end{array}\right)\left(\begin{array}{c}
c+l \\
l
\end{array}\right)} \\
& \quad=\sum_{n=0}^{\infty}(-1)^{n}\left(\begin{array}{c}
n+m-1 \\
n
\end{array}\right) \frac{a b c n^{3} \Gamma(a n) \Gamma(j+1) \Gamma(b n) \Gamma(k+1) \Gamma(c n) \Gamma(l+1)}{\Gamma(a n+j+1) \Gamma(b n+k+1) \Gamma(c n+l+1)} \\
& =\sum_{n=0}^{\infty}(-1)^{n} a b c n^{3}\left(\begin{array}{c}
n+m-1 \\
n
\end{array}\right) B(a n, j+1) B(b n, k+1) B(c n, l+1) \\
& =1+a b c \sum_{n=1}^{\infty}(-1)^{n} n^{3}\left(\begin{array}{c}
n+m-1 \\
n
\end{array}\right) \int_{0}^{1}(1-x)^{j-1} x^{a n} d x \int_{0}^{1}(1-y)^{k-1} y^{b n} d y \int_{0}^{1}(1-z)^{l-1} z^{c n} d z .
\end{aligned}
$$

By an allowable change of integral and sum, we have

$$
\begin{aligned}
& R(a, b, c, j, k, l, m) \\
& \quad=1+a b c \iiint_{0}^{1} \frac{(1-x)^{j}(1-y)^{k}(1-z)^{l}}{x y z} \sum_{n=1}^{\infty}(-1)^{n}\left(\begin{array}{c}
n+m-1 \\
m-1
\end{array}\right) n^{3}\left(x^{a} y^{b} z^{c}\right)^{n} d x d y d z \\
& \quad=1-m a b c \iiint_{0}^{1}(1-x)^{j}(1-y)^{k}(1-z)^{l} \frac{X Y Z\left((X Y Z)^{2}-(3 m+1) X Y Z+1\right)}{x y z(1+X Y Z)^{m+3}} d x d y d z
\end{aligned}
$$

which is the result (3.17).

To arrive at the result (3.16), consider

$$
\begin{aligned}
& R(a, b, j, c, k, l, m) \\
& \quad=j k l \sum_{n=0}^{\infty}(-1)^{n}\left(\begin{array}{c}
n+m-1 \\
n
\end{array}\right) \frac{\Gamma(a n+1) \Gamma(j) \Gamma(b n+1) \Gamma(k) \Gamma(c n+1) \Gamma(l)}{\Gamma(a n+j+1) \Gamma(b n+k+1) \Gamma(c n+l+1)} \\
& \quad=j k l \sum_{n=0}^{\infty}(-1)^{n}\left(\begin{array}{c}
n+m-1 \\
n
\end{array}\right) B(a n+1, j) B(b n+1, k) B(c n+1, l) \\
& \quad=j k l \sum_{n=0}^{\infty}(-1)^{n}\left(\begin{array}{c}
n+m-1 \\
n
\end{array}\right) \int_{0}^{1}(1-x)^{j-1} x^{a n} d x \int_{0}^{1}(1-y)^{k-1} y^{b n} d y \int_{0}^{1}(1-z)^{l-1} z^{c n} d z \\
& \quad=j k l \iiint_{0}^{1}(1-x)^{j-1}(1-y)^{k-1}(1-z)^{l-1} \sum_{n=0}^{\infty}(-1)^{n}\left(\begin{array}{c}
n+m-1 \\
n
\end{array}\right)\left(x^{a} y^{b} z^{c}\right)^{n} d x d y d z
\end{aligned}
$$


by an allowable change of sum and integral, hence

$$
R(a, b, j, c, k, l, m)=j k l \iiint_{0}^{1} \frac{(1-x)^{j-1}(1-y)^{k-1}(1-z)^{l-1}}{(1+X Y Z)^{m}} d x d y d z
$$

which is the result (3.16).

The hypergeometric representations (3.13) and (3.18) can be obtained by the consideration of the ratio of successive terms (3.9) and (3.14), respectively.

In the case when $m=1$, Theorem 3.5 reduces to Theorem 2.1 .

\section{Examples}

Example 4.1. It holds that

$$
\begin{aligned}
& Q(4,3,2,5,3,6,11) \\
& =\sum_{n=0}^{\infty} \frac{\left(\begin{array}{c}
n+10 \\
n
\end{array}\right)}{\left(\begin{array}{c}
4 n+5 \\
5
\end{array}\right)\left(\begin{array}{c}
3 n+3 \\
3
\end{array}\right)\left(\begin{array}{c}
2 n+6 \\
6
\end{array}\right)} \\
& =90 \iiint_{0}^{1} \frac{(1-x)^{4}(1-y)^{2}(1-z)^{5}}{\left(1-x^{4} y^{3} z^{2}\right)^{11}} d x d y d z \\
& =1+264 \iiint_{0}^{1} \frac{(1-x)^{5}(1-y)^{3}(1-z)^{6} x^{3} y^{2} z\left(x^{8} y^{6} z^{4}+34 x^{4} y^{3} z^{2}+1\right) d x d y d z}{\left(1-x^{4} y^{3} z^{2}\right)^{14}} \\
& ={ }_{10} F_{9}\left[\begin{array}{c}
11,1,1,1, \frac{3}{4}, \frac{2}{3}, \frac{1}{2}, \frac{1}{2}, \frac{1}{3}, \frac{1}{4} \\
4, \frac{7}{2}, \frac{9}{4}, 2,2, \frac{5}{3}, \frac{7}{4}, \frac{3}{2}, \frac{4}{3}
\end{array} \mid 1\right] \\
& =\frac{3413 \cdot 43 \cdot 5 \cdot 3}{2^{14}} \zeta(2)+\left(\frac{1931 \cdot 509}{2^{12}}-\frac{90379 \sqrt{3}}{7 \cdot 3 \cdot 2^{5}}\right) \pi-\frac{1459 \cdot 1231}{7 \cdot 3^{2} \cdot 2^{11}} \\
& -\frac{379880779}{7 \cdot 3 \cdot 2^{10}} \ln 2+\frac{22567 \cdot 3^{2}}{7 \cdot 2^{5}} \ln 3 .
\end{aligned}
$$

Example 4.2. It holds that

$$
\begin{aligned}
R(1 / 4,1 / 6,1 / 2,5,3,7,14) & :=\sum_{n=0}^{\infty} \frac{(-1)^{n}\left(\begin{array}{c}
n+13 \\
n
\end{array}\right)}{\left(\begin{array}{c}
n / 4+5 \\
5
\end{array}\right)\left(\begin{array}{c}
n / 6+3 \\
3
\end{array}\right)\left(\begin{array}{c}
n / 2+7 \\
7
\end{array}\right)} \\
& =\sum_{n=0}^{\infty} \frac{(-1)^{n} 5 ! 3 ! 7 !(n+1)_{13}}{13 !(n / 4+1)_{5}(n / 6+1)_{3}(n / 2+1)_{7}} \\
& =105 \iiint_{0}^{1} \frac{(1-x)^{4}(1-y)^{2}(1-z)^{6}}{\left(1+x^{1 / 4} y^{1 / 6} z^{1 / 2}\right)^{14}} d x d y d z
\end{aligned}
$$




$$
\begin{aligned}
& =1+\frac{7}{24} \iiint_{0}^{1}(1-x)^{5}(1-y)^{3}(1-z)^{7} \frac{\left(x^{1 / 2} y^{1 / 3} z+43 x^{1 / 4} y^{1 / 6} z^{1 / 2}+1\right)}{x^{3 / 4} y^{5 / 6} z^{1 / 2}\left(1-x^{1 / 4} y^{1 / 6} z^{1 / 2}\right)^{17}} d x d y d z \\
& ={ }_{16} F_{15}\left[\begin{array}{c}
2,4,4,6,6,8,8,10,12,12,12,14,14,16,18,20 \\
3,5,5,7,7,9,9,11,13,13,13,15,17,19,21
\end{array} \mid-1\right] \\
& =\frac{7 \cdot 5 \cdot 3^{3} \cdot 2^{4}}{13} \zeta(2)-\frac{16231 \cdot 11 \cdot 3}{7 \cdot 5 \cdot 2^{3}} .
\end{aligned}
$$

\section{Conclusion}

We have provided triple integral identities for sums of the reciprocal of triple binomial coefficients. In doing so, we have recovered the standard representation for $\zeta(3)$ and have generalised and extended some results published previously by other authors.

In another forum, we will extend our results to consider binomial coefficients of the form

$$
\sum_{n=0}^{\infty} \frac{n^{s}\left(\begin{array}{c}
n+m-1 \\
n
\end{array}\right)}{\left(\begin{array}{c}
a n+j \\
j
\end{array}\right)\left(\begin{array}{c}
b n+k \\
k
\end{array}\right)\left(\begin{array}{c}
c n+l \\
l
\end{array}\right)}, \quad \sum_{n=0}^{\infty} \frac{\left(\begin{array}{c}
n+m-1 \\
n
\end{array}\right)}{\left(\begin{array}{c}
a n+j \\
b n
\end{array}\right)\left(\begin{array}{c}
c n+k \\
d n
\end{array}\right)\left(\begin{array}{c}
p n+j \\
q n
\end{array}\right)} .
$$

\section{Acknowledgment}

This paper was completed while the author was a Visiting Professor at the Dipartimento di Sistemi e Informatica, Universita di Firenze. I wish to express my sincere thanks to Professor Sprugnoli for his hospitality.

\section{References}

[1] A. Sofo, "General properties involving reciprocals of binomial coefficients," Journal of Integer Sequences, vol. 9, no. 4, Article ID 06.4.5, 13 pages, 2006.

[2] A. Sofo, Computational Techniques for the Summation of Series, Kluwer Academic/Plenum Publishers, New York, NY, USA, 2003.

[3] A. Sofo, "Integral representations of ratios of binomial coefficients," International Journal of Pure and Applied Mathematics, vol. 31, no. 1, pp. 29-46, 2006.

[4] A. Sofo, "Some properties of reciprocals of double binomial coefficients," accepted.

[5] http://mathworld.wolfram.com/RiemannZetaFunction.html.

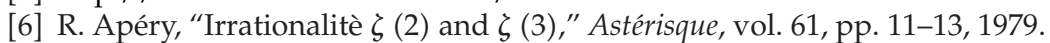

[7] F. Beukers, "A note on the irrationality of $\zeta(2)$ and $\zeta(3)$, , Bulletin of the London Mathematical Society, vol. 11, no. 3, pp. 268-272, 1979 .

[8] J. Guillera and J. Sondow, "Double integrals and infinite products for some classical constants via analytic continuations of Lerch's transcendent," to appear in The Ramanujan Journal.

[9] H. Muzaffar, "Some interesting series arising from the power series expansion of $\left(\sin ^{-1} x\right)^{q}$," International Journal of Mathematics and Mathematical Sciences, vol. 2005, no. 14, pp. 2329-2336, 2005.

[10] S. B. Ekhad and D. Zeilberger, "A 21st century proof of Dougall's hypergeometric sum identity," Journal of Mathematical Analysis and Applications, vol. 147, no. 2, pp. 610-611, 1990.

[11] L. J. Slater, Generalized Hypergeometric Functions, Cambridge University Press, Cambridge, UK, 1966.

[12] S. R. Finch, Mathematical Constants, vol. 94 of Encyclopedia of Mathematics and Its Applications, Cambridge University Press, Cambridge, UK, 2003.

[13] N. Lord, "Problem corner," The Mathematical Gazette, vol. 89, no. 514, pp. 115-119, 2005.

[14] Z. Nan-Yue and K. S. Williams, "Values of the Riemann zeta function and integrals involving $\log (2 \sinh (\theta / 2))$ and $\log (2 \sin (\theta / 2)), "$ Pacific Journal of Mathematics, vol. 168, no. 2, pp. 271-289, 1995. 


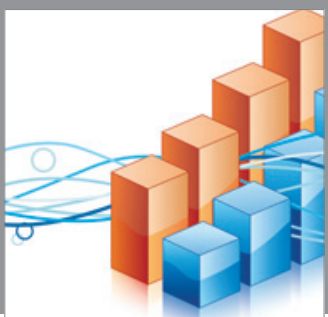

Advances in

Operations Research

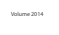

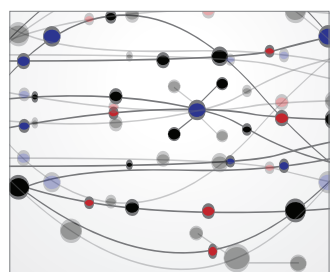

\section{The Scientific} World Journal
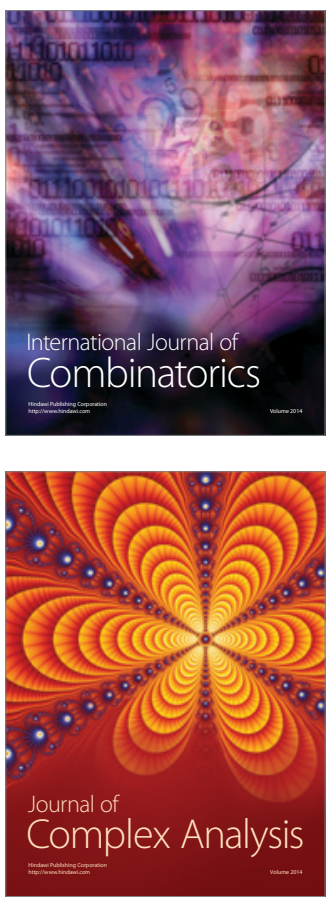

International Journal of

Mathematics and

Mathematical

Sciences
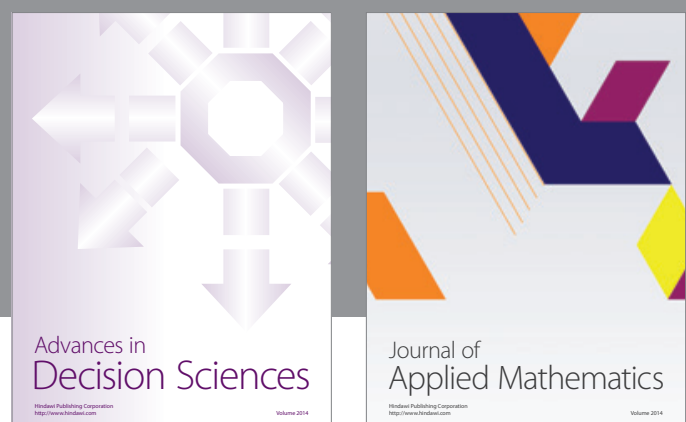

Journal of

Applied Mathematics
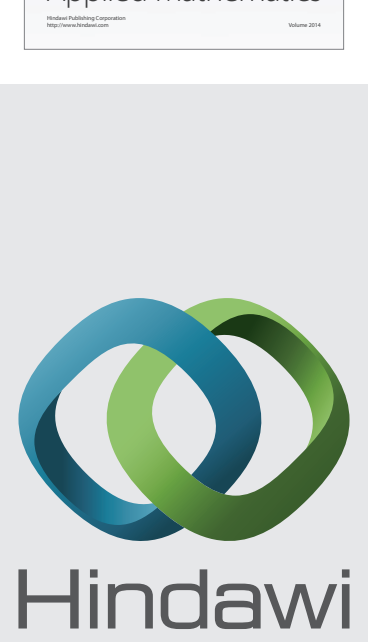

Submit your manuscripts at http://www.hindawi.com
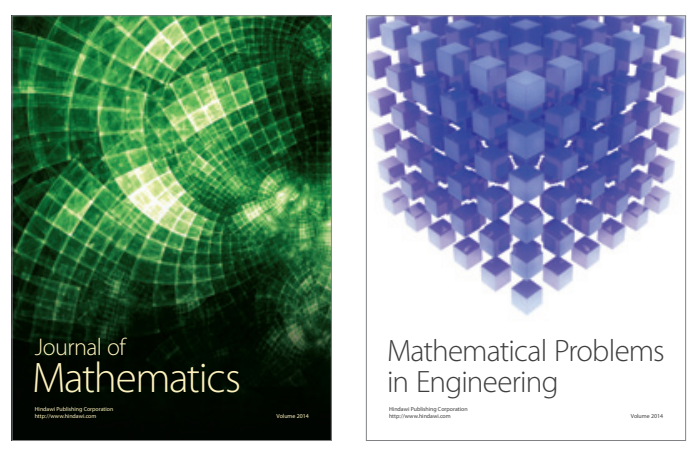

Mathematical Problems in Engineering
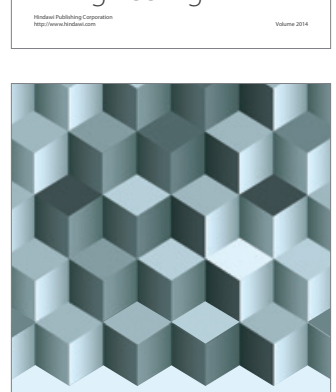

Journal of

Function Spaces
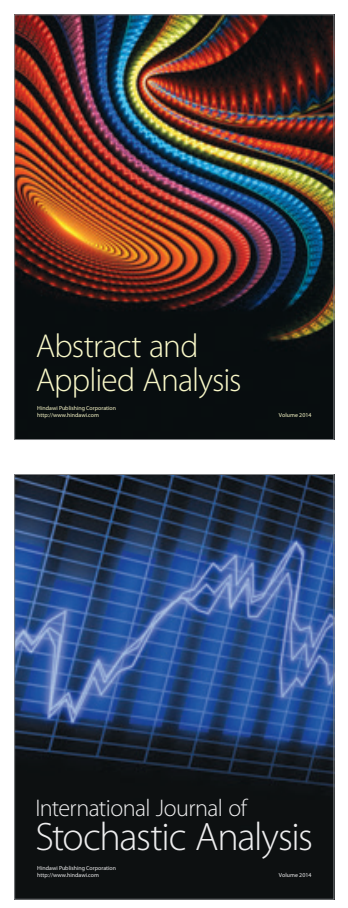

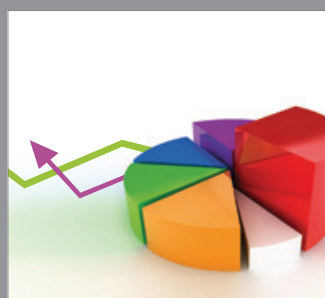

ournal of

Probability and Statistics

Promensencen
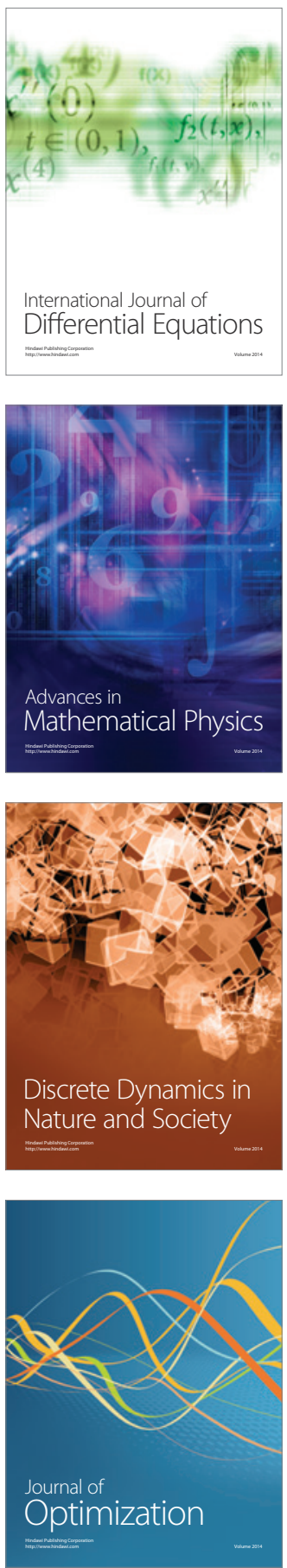